\title{
Pre-imaginal conditioning alters adult sex pheromone response in Drosophila
}

\author{
Claude Everaerts ${ }^{\text {Corresp.., }}{ }^{1}$, Laurie Cazalé-Debat ${ }^{1}$, Alexis Louis ${ }^{1}$, Emilie Pereira ${ }^{1}$, Jean-Pierre Farine ${ }^{1}$, \\ Matthew Cobb ${ }^{2}$, Jean-François Ferveur ${ }^{1}$ \\ ${ }^{1}$ Centre des Sciences du Goût et de I'Alimentation, Agrosup-UMR 6265 CNRS, UMR 1324 INRA, Université de Bourgogne, Dijon, France \\ 2 School of Biological Sciences, University of Manchester, Manchester, United Kingdom \\ Corresponding Author: Claude Everaerts \\ Email address: Claude.Everaerts@u-bourgogne.fr
}

Pheromones are chemical signals that induce innate responses in individuals of the same species that may vary with physiological and developmental state. In Drosophila melanogaster, the most intensively studied pheromone is 11-cis-vaccenyl acetate (cVA), which is synthezised in the male ejaculatory bulb and is transferred to the female during copulation. Among other effects, cVA inhibits male courtship of mated females. We found that male courtship inhibition depends on the amount of CVA and this effect is reduced in male flies derived from eggs covered with low to zero levels of cVA. This effect is not observed if the eggs are washed, or if the eggs are laid several days after copulation. This suggests that courtship suppression involves a form of pre-imaginal conditioning, which we show occurs during the early larval stage. The conditioning effect could not be rescued by synthetic CVA, indicating that it largely depends on conditioning by CVA and other maternally-transmitted factor(s). These experiments suggest that one of the primary behavioral effects of CVA is more plastic and less stereotypical than had hitherto been realised. 
1

2

3

4

5

6

7

8

9

10

11

12

13

$14{ }^{1}$ Centre des Sciences du Goût et de l'Alimentation, AgroSup Dijon, CNRS, INRA, 15 Univ. Bourgogne Franche-Comté, F-21000 Dijon, France

162 School of Biological Sciences, University of Manchester, Oxford Road, Manchester

17 M13 9PT, UK.

18

19 Corresponding author:

20 Claude Everaerts

216 bd. Gabriel, F-21000 Dijon, France

22 Claude.Everaerts@u-bourgogne.f 


\section{ABSTRACT}

25

26 Pheromones are chemical signals that induce innate responses in individuals of the 27 same species that may vary with physiological and developmental state. In Drosophila 28 melanogaster, the most intensively studied pheromone is 11-cis-vaccenyl acetate 29 (cVA), which is synthezised in the male ejaculatory bulb and is transferred to the female 30 during copulation. Among other effects, cVA inhibits male courtship of mated females. 31 We found that male courtship inhibition depends on the amount of cVA and this effect is 32 reduced in male flies derived from eggs covered with low to zero levels of cVA. This 33 effect is not observed if the eggs are washed, or if the eggs are laid several days after 34 copulation. This suggests that courtship suppression involves a form of pre-imaginal conditioning, which we show occurs during the early larval stage. The conditioning 36 effect could not be rescued by synthetic cVA, indicating that it largely depends on 37 conditioning by $\mathrm{cVA}$ and other maternally-transmitted factor(s). These experiments 38 suggest that one of the primary behavioral effects of cVA is more plastic and less 39 stereotypical than had hitherto been realised. 


\section{INTRODUCTION}

41

42 Pheromones, first identified as chemicals that release a certain behaviour or 43 physiological response (Karlson \& Butenandt 1959) have recently been more precisely defined as chemical signals that induce innate stereotypical responses in individuals of the same species (Wyatt 2015). However, the way that pheromones act can depend on the physiological and developmental state of the individual that receives them. One of the most intensely studied pheromones is the Drosophila sex pheromone, 11-cisvaccenyl acetate (cVA). This compound induces sex-specific effects when it is transferred from males to females during mating, and then introduced into the food substrate during egg-laying. This lipid-derived compound, which is produced in the male ejaculatory bulb, inhibits male courtship of mated females, renders food more attractive to males and females, stimulates females to mate and plays a role in inducing malemale aggression (Bartelt et al. 1985a; Butterworth 1969; Das et al. 2017; Ejima 2015; Fernandez \& Kravitz 2013; Guiraudie-Capraz et al. 2007; Jallon et al. 1981; Kurtovic et al. 2007; Laturney \& Billeter 2016; Lebreton et al. 2015; Schaner et al. 1987; Wang et al. 2011; Wertheim et al. 2005; Zawistowski \& Richmond 1986).

Recently, the neuronal circuits involved in processing cVA and producing an appropriate behavioral output have been explored, giving some indication of the circuitry involved in the sex-specific effects. In both sexes, cVA is detected by the antennal sensilla expressing the olfactory receptors Or65a and Or67d (Clyne et al. 1997; van der Goes van Naters \& Carlson 2007). These neurons project to the DL3 and DA1 glomeruli respectively (Couto et al. 2005; Fishilevich et al. 2005; Lebreton et al. 2015). Projection neurons leaving DA1 show sexually dimorphic arborization in higher brain centers (Datta et al. 2008; Kohl et al. 2013; Ruta et al. 2010). In males, stimulation by cVA together with the male-specific cuticular hydrocarbons leads to male-male aggression (Fernandez et al. 2010). In both sexes, chronic stimulation by cVA activates the DL3 glomerulus which then inhibits the output of the DA1 glomerulus via the activity of an inhibitory lateral interneuron. In the male, this leads to courtship suppression (Kurtovic 
70 et al. 2007), whereas in the female it leads to a decline in attraction to cVA (Lebreton et 71 al. 2015).

Common to all these studies is the assumption that responses to cVA are stereotypic and unconditional. Although some studies have described a variable effect of cVA in a early adult conditioning (Liu et al. 2011; Tachibana et al. 2015), nothing is known about the potential conditioning effect of cVA during pre-imaginal development (after 'imago', the technical term for the adult insect). The term 'conditioning' has two meanings in the literature: it can mean treating an animal at one stage with a particular stimulus, and then observing the consequences of that treatment, irrespective of the mechanism involved; this is the sense in which we employ the term here, and is congruent with the earliest studies of changes in Drosophila behaviour following early experience (Manning 1967; Thorpe 1939), in which the effect was called pre-imaginal conditioning. The second, more specific use of the term relates to particular models of associative learning (e.g. classical or operant conditioning); we have not investigated any form of learning here, and do not use the term 'conditioning' in this sense.

Studying the robust and well-known male courtship suppression phenomenon, we show that adult male responses to cVA are a consequence of exposure to a combination of substances, including cVA, during the larval stage. Furthermore, there is natural variability for this conditioning effect. This finding explains inter-individual response variability to cVA and opens the road to the study of how pre-imaginal conditioning affects nervous system development, and its evolutionary significance.

\section{METHODS}

\section{Flies}

We used the D. melanogaster wild-type stock Dijon2000 (Di2 (Houot et al. 2010); WT1) and the Di2/w ${ }^{1118}$ line (WT2), derived from the Di2 strain in which we introgressed the genome of the $w^{1118}$ strain over five repeated backcross generations. This white-eyed line was used to allow us to distinguish adults from this strain and those from the 
101 conditioned strain (which had red eyes). No effect due to the mutation was observed.

102 Stocks were raised on yeast / cornmeal / agar medium (6,5 L distilled water, $425 \mathrm{~g}$ 103 maize flour, $425 \mathrm{~g}$ beer yeast, $60 \mathrm{~g}$ agar and $200 \mathrm{ml}$ of $1 \%$ solution of nipagin, Sigma 104 Aldrich, diluted in ethanol). Flies were kept at $24 \pm 0.5^{\circ}$ and $65 \pm 5 \%$ humidity on a $12 \mathrm{~L}$ : $10512 \mathrm{D}$ cycle (subjective day=8:00 am to 8:00 pm) and were isolated under light $\mathrm{CO}_{2}$ 106 anaesthesia either $0-4 \mathrm{~h}$ (for virgin females) or less than $24 \mathrm{~h}$ (for virgin males) after 107 eclosion. Adult flies were held for 4 days in $30 \mathrm{ml}$ glass vials filled with $4 \mathrm{ml}$ fresh plain

108 food. Same sex flies were always kept in small groups except for focal males used in 109 behavioral tests; these were isolated to avoid social interactions affecting their 110 subsequent courtship behavior (Svetec \& Ferveur 2005).

\section{Egg collection and treatment}

1131 hour after subjective dawn, 30 male flies and 10 females, all 4-day-old, were placed in 114 a $30 \mathrm{ml}$ glass vial containing $4 \mathrm{ml}$ fresh plain food. After $3 \mathrm{~h}$, flies were cold-anaesthetized $115\left(15 \mathrm{~min}\right.$ at $\left.4^{\circ} \mathrm{C}\right)$ and females were transferred into egg-laying devices. Each device 116 consisted of a $50 \mathrm{~mm}$ Petri dish filled with $1 \mathrm{ml} \mathrm{3 \%}$ agar striped with fresh yeast to 117 stimulate egg-laying. Cold-anaesthetized mated females were aspirated into the egg118 laying device through a small hole on the top of the Petri dish which was closed with a 119 small metal plug. Three hours later, females were discarded and eggs collected. In the 120 case of flies used to produce eggs at 10 days after mating, the females were placed in a 121 tube until the appropriate time.

123 In all control experiments, and when indicated, focal males resulted from eggs laid by 124 females $24 \mathrm{~h}$ after mating (+1D), except where focal males originated from eggs laid by

125 the same females 10 days after mating (+10D) (Fig. 1). In some cases, eggs were 126 washed just after collection with a $10 \%$ ethanol solution (10\%) to remove cVA and other 127 chemicals from the egg surface ("Wash"). Some Wash eggs were submitted to 128 additional treatments, either soaked in a synthetic cVA solution (100ng cVA/ $\mu$ l pure 129 water; Cayman Chemical ; $50 \mathrm{mg} / \mathrm{ml}$ solution in éthanol ; purity $>98 \%$ ) for $5 \mathrm{~min}$, 130 deposited on food enriched with cVA $\left(15 \mathrm{ng} / \mathrm{mm}^{3}\right)$, or on food seeded with eggs laid by 131 WT2 females $24 \mathrm{~h}$ after mating with conspecific males. WT2 flies were discarded at 
132 emergence (based on their white eye color) to leave WT1 flies. To determine the critical

133 period for conditioning, eggs (or larvae) were collected and washed with $10 \%$ ethanol

134 solution at $10,20,40$ or $72 \mathrm{~h}$ after egg-laying. In all control and experimental tests, Wash

135 eggs (or larvae) were placed in groups of 50 on the food (plain or CVA-rich) contained in 136 glass vials.

\section{Behavior}

139 Less than $24 \mathrm{~h}$ after eclosion, focal males were screened under light $\mathrm{CO}_{2}$ anaesthesia 140 and individually placed in $30 \mathrm{ml}$ glass vials containing $4 \mathrm{ml}$ fresh plain food until the test

141 (4-days old). Behavioral tests were performed under white light in a specially designed 142 mating chamber consisting of two superposed parts (Diameter $=11 \mathrm{~mm}$; height $=5 \mathrm{~mm}$ ) 143 separated by a fine nylon mesh (Fig.1). The lower part contained a filter paper (25 $\left.\mathrm{mm}^{2}\right)$ 144 impregnated with a controlled dose of cVA and small amount of yeast (ca. $125 \mathrm{~mm}^{3}$ ). A 145 single focal male was aspirated into the top part, then after $10 \mathrm{~min}$ acclimation a 4-day146 old virgin female that had previously been decapitated was aspirated in the top part. We 147 did not use mated females, because they are expected to carry cVA following mating, 148 and the exact amount would vary between females. Virgin $\mathrm{CO}_{2}$-anaesthetized females 149 were decapitated with a razor blade, $1 \mathrm{~h}$ before the test. As in many previous studies, we 150 used decapitated virgin females as the target fly; these remained alive, but mostly 151 immobile, for at least 3-4h. Decapitated virgin females do not produce rejection signals 152 and do not copulate: this allows us to eliminate most female-produced signals that might 153 otherwise bias measures of male activity. Decapitated females were therefore live targets 154 producing stimulatory signals that enabled us to measure the inhibitory effect induced by cVA on 155 male excitation. Total male activity was noted for $10 \mathrm{~min}$, including courtship latency (time 156 from introduction to courtship onset) and the total percentage of time spent courting 157 (courtship index : $\mathrm{Cl}$ ). $\mathrm{Cl}$ consists of the cumulative duration of all courtship behaviors 158 shown by the subject male (Greenspan \& Ferveur 2000; Lasbleiz et al. 2006). To 159 estimate the frequency of male courtship we counted the number of males showing a $160 \mathrm{Cl}>5$ divided by the total number of males tested. Males from all treatments were 161 always tested in parallel with control males (dervied from sham-control eggs that had 162 been handled, but were otherwise untreated) and in some cases with males derived 
163 from washed eggs. The experiments were conducted by individual observers who were 164 unaware of what treatment the flies had been subjected to.

165

\section{Determination of cVA levels}

167 To quantify the amount of cVA on 4-day-old individual flies, we immersed frozen flies $168\left(5 \mathrm{~min}\right.$ at $\left.-20^{\circ} \mathrm{C}\right)$ for $5 \mathrm{~min}$ at room temperature in $30 \mu \mathrm{l}$ of a hexane solution containing $1693.33 \mathrm{ng} / \mathrm{\mu l}$ of two internal standards (n-hexacosane and $\mathrm{n}$-triacontane). We used a 170 similar procedure to determine the amount of cVA in groups of 50 eggs. To evaluate the 171 internal amount of cVA, frozen individuals were soaked in a similar hexane solution for $17224 \mathrm{~h}$ at $40^{\circ} \mathrm{C}$ (Bartelt et al. 1985b). The resulting extract and two successive rinses of 173 each individual fly were combined and reduced in volume, under $\mathrm{N}_{2}$ flow, for 174 chromatographic analysis. cVA was quantified by gas chromatography using a Varian 175 CP3380 gas chromatograph fitted with a flame ionization detector, a CP Sil 5CB column 176 (25mx0.25 mm internal diameter; $0.1 \mu \mathrm{m}$ film thickness; Agilent), and a split-splitless 177 injector (60 $\mathrm{ml} / \mathrm{min}$ split-flow; valve opening $30 \mathrm{sec}$ after injection) with helium as carrier 178 gas (velocity $=50 \mathrm{~cm} / \mathrm{sec}$ at $120^{\circ} \mathrm{C}$ ). The temperature program began at $120^{\circ} \mathrm{C}$, ramping 179 at $10^{\circ} \mathrm{C} / \mathrm{min}$ to $140^{\circ} \mathrm{C}$, then ramping at $2^{\circ} \mathrm{C} / \mathrm{min}$ to $290^{\circ} \mathrm{C}$, and holding for $10 \mathrm{~min}$. The 180 chemical identity of cVA was checked using gas chromatography - mass spectrometry 181 equipped with a CP Sil 5CB column. The amount (in ng) of cVA was calculated on the 182 basis of the data obtained from the internal standards.

183

184 Statistics

185 All statistical analyses were performed using XLSTAT 2012 (2012). We used logistic 186 regression to characterize the relationship between cVA amount and courtship inhibition 187 by estimating the active dose 50 (AD50) (Robertson \& Preisler 1992). Courtship 188 frequencies were compared using a Wilks $G^{2}$ likelihood ratio test completed with a 189 computation of significance by cell (Fisher's test). Comparisons of courtship indices, 190 courtship latencies or cVA levels were carried out either with a Kruskall-Wallis test with 191 Conover-Iman multiple pairwise comparisons ( $p=0.05$, with a Bonferroni correction) or 192 with a Mann-Whitney test, after excluding extreme outliers using Tukey's method 193 (Tukey 1977). 


\section{RESULTS}

195

196

197

198

199

200

201

202

203

204

205

206

207

208

209

210

211

212

213

214

215

216

217

218

219

220

221

222

223

224

Measurable amounts of cVA are transferred to eggs laid one day after mating $(0.21 \pm 0.02 \mathrm{ng} ;+1 \mathrm{D})$ and can be completely eliminated by washing the eggs with a $10 \%$ ethanol solution (Ong; Fig.2). Males derived from control, unwashed eggs showed a clear dose-response courtship suppression effect when their courtship towards an immobilised virgin female was observed in a chamber placed above a source of varying amounts of cVA (Fig.1). Both courtship frequency $\left(\mathrm{Khi}^{2}{ }_{9 \mathrm{df}}=89.4, p<10^{-4}\right.$; filled bars in Fig.3a) and courtship index (Cl; $\mathrm{KW}_{9 \mathrm{df}}=129.4, p<10^{-4}$; filled circles in Fig.3b) showed clear negative correlations with cVA levels. When no cVA was present below the mating chamber, control males courted females with a high frequency (85\%) and a strong courtship index $(\mathrm{Cl}=0.34 \pm 0.02)$; in the presence of $600 \mathrm{ng}$ cVA these figures declined to $40 \%$ courtship and a $\mathrm{Cl}=0.07 \pm 0.02$. Maximum courtship suppression was induced with 600ng, and 50\% inhibition was observed with 350ng; subsequent tests were performed with these two cVA doses. These levels are of the same order of magnitude as the total amount of cVA present in male flies (974 $186 \mathrm{ng}$; Fig.S1), or on the cuticle of a mated female (313 $\pm 23 n g)$. Virtually no cVA can be detected on the cuticle of a virgin female $(0.4 \pm 0.04 \mathrm{ng})$.

Strikingly, if cVA was removed from eggs by washing them immediately after laying, males derived from these eggs showed no dose-response courtship suppression, even with very high cVA doses (e.g. 2000ng; frequency $=78 \%$; $\mathrm{Cl}=0.31 \pm 0.02$; empty bars and diamonds in Fig.3a,b). Egg-washing had no effect on male behavior (Fig.3d). This suggests that cVA-based courtship suppression is produced through a pre-imaginal conditioning effect on the developing fly. Variation in the amount of cVA present on eggs occurs naturally, as revealed by a comparison of eggs laid by the same females one day (+1D) and 10 days (+10D) after mating. At $+10 D$, eggs were covered in no detectable cVA (Ong, as compared to $0.25 \pm 0.03$ in $+1 \mathrm{D}$ flies; $\cup_{12,10}=120$, $p=0.005$; Fig. 4a). Males derived from $+10 \mathrm{D}$ eggs showed no courtship suppression $\left(\mathrm{U}_{10,8}=35\right.$, $p=0.70$; Figs. $2 \mathrm{~b}$ and S2), unlike males derived from +1D eggs, which showed significantly less intense courtship in the presence of cVA $\left(\cup_{19,14}=209, p=0.005\right)$. Taken 
225 together, these data show that male courtship suppression by cVA involves a

226 conditioning effect that is mediated by the presence of a substance or substances on 227 eggs which is sex-peptide ed with the level of cVA. To reveal whether there is a critical 228 period for conditioning, eggs were washed at different periods after laying (10h, 20h), 229 and larvae were washed $48 \mathrm{~h}$ and $72 \mathrm{~h}$ after egg-laying; Fig. 5). No courtship 230 suppression was observed if eggs were washed $10 \mathrm{~h}$ or $20 \mathrm{~h}$ after egg laying, while 231 control levels of suppression (indicating the existence of pre-imaginal conditioning) were 232 seen when older larvae were washed (48h or $72 \mathrm{~h}$ after egg-laying) $\quad\left(\mathrm{KW}_{4 \mathrm{df}}=15.6\right.$; $233 \mathrm{p}=0.004$; Figs. 5a and S3a. No effect of egg wash was detected in the absence of cVA 234 below the courtship chamber (Figs.5b and S3b).

236 We were able to rescue courtship suppression by placing washed eggs on food 237 containing unwashed eggs (and therefore natural levels of female-deposited 238 compounds). Males derived from these eggs showed a courtship suppression similar to 239 control males (frequency $=45 \%, \mathrm{Khi}^{2}{ }_{7 \mathrm{df}}=55.5, p<10^{-4} ; \mathrm{Cl}=0.22 \pm 0.045 ; \mathrm{KW}_{7 \mathrm{df}}=34.3, p<10^{-}$ 240 4; light gray bars in Figs.3c,d). This shows that an apparent critical period for pre241 imaginal conditioning occurs after larval eclosion from the egg, which occurs around 2024224 hours after egg-laying. This may be a true critical period requiring the nervous 243 system to be in a particular state, or it may simply reveal that pre-imaginal conditioning 244 requires the larva to come into contact with substances on the outside of the egg.

246 However, when we placed washed eggs on food enriched with synthetic cVA $247\left(15 \mathrm{ng} / \mathrm{mm}^{3}\right)$, males derived from these eggs showed no courtship suppression 248 (frequency $=75 \%$; $\mathrm{Cl}=0.53 \pm 0.06$; dark gray bars in Figs.3c,d and S4); similar results 249 were obtained when washed eggs were covered with synthetic cVA (Fig. 6). Although 250 the amount of synthetic cVA recovered from eggs in these two experiments was similar 251 to the levels of natural cVA found on control eggs $(0.29 \pm 0.05$ and $0.21 \pm 0.02 \mathrm{ng}$, 252 respectively, $P=0.126$; Fig.6a), both of these sets of males behaved like males derived 253 from washed eggs (Fig.6b, Fig.S5). Assuming that synthetic cVA is chemically identical 254 to its natural equivalent, we conclude that other compounds coating the egg, or forming 
255 the egg coat, are at least partly responsible for the larval conditioning effect on cVA256 mediated courtship suppression, probably in synergy with cVA. 


\section{DISCUSSION}

258

259 The rich behavioral effects induced by cVA in male and female flies have become the

260 focus of a series of neurobiological studies that have begun to reveal the neuronal

261 circuitry involved. Here we show that the longest-established of these effects - male

262 courtship suppression - is a conditional phenotype, dependent not only upon the

263 apparent learning effect occurring during adult life (Siegel \& Hall 1979) but also on

264 exposure to the substance during the first instar larval stage - pre-imaginal conditioning.

265 In addition to an effect imputable to cVA, this conditioning is induced by unknown stimuli

266 that are introduced onto the egg by the female, or which are present on the chorion and

267 are detected by the larva on eclosion. This conclusion is based on the following results:

268 the effect is blocked by washing the eggs, can be rescued by putting washed eggs on

269 food containing normal eggs, but cannot be rescued by adding cVA alone (Figs.3c,d).

271 The pre-imaginal conditioning described here appears to occur in the first larval stage -

272 washing of eggs at $10 \mathrm{~h}$ and $20 \mathrm{~h}$ after egg-laying prevented conditioning as shown by

273 the abolition of cVA-based courtship suppression, while washing of larvae at $48 \mathrm{~h}$ or $72 \mathrm{~h}$

274 after egg-laying had no effect on courtship suppression, revealing control levels of

275 conditioning. Moreover, males derived from washed eggs deposited on food containing

276 control eggs also showed courtship suppression, suggesting that pre-imaginal

277 conditioning does not occur in the egg itself but immediately after eclosion of the first

278 instar larva.

279

280 The absence of courtship suppression in males derived from washed eggs reared in the

281 presence of synthetic cVA could be taken to indicate that cVA is not involved at all in

282 this effect. However, this would be to overlook the clear dose-response effect of cVA

283 shown on normal males in the presence of cVA, which demonstrates a role for cVA in

284 courtship suppression. We conclude that the simultaneous presence of another factor or

285 factors, along with cVA, is required during the conditioning exposure phase to induce

286 the modulation of cVA perception in adult males. Washing the eggs removed both cVA

287 and these unknown factors. Covering washed eggs with synthetic cVA did not rescue 
288 the adult response to cVA because those other factor(s) are necessary to create an

289 association or synergy with cVA.

290

291 A diverse range of substances, unmeasured in our experiments, could conceivable act

292 additively with cVA: for example, sex-peptides which are transferred during mating and

293 disappear from the female genital tract after several days (Peng et al. 2005),

294 metabolites produced by the microbes present on the chorion and which disappear after

295 egg-washing (Farine et al. 2017), or cuticular hydrocarbons which are found in trace

296 levels on eggs, but which not no detectable variation with female age post-mating (total

$297 \mathrm{CHs}$ amount $=11.9 \pm 0.8$ at $\mathrm{D} 1$ and $13.9 \pm 1.4$ at $\mathrm{D} 10 ; \mathrm{U}_{10,10}=32, p=0.186$; Fig. S7).

298 Whatever the case, our data firmly indicate that cVA is involved since we measured and 299 compared the adult male response in the presence/absence of this substance only. It is

300 also possible that the synthetic cVA that we used does not exactly cover the chorion

301 structure as in the natural situation.

302

303 This effect occurs pre-pupation, whereas the phenotype we have studied is expressed

304 in the adult. Changes to adult behavior consequent on larval experience - generally

305 involving olfaction - have been widely reported in Drosophila and in other

306 holometabolous insects, going back to the 1930s and are known as pre-imaginal

307 conditioning (Manning 1967; Thorpe 1939) for a review see (Barron 2001; Barron \&

308 Corbet 1999). This phenomenon is distinct from the sparse claims for transfer of

309 memory through pupation in holometabolous insects (e.g. Tully et al. 1994). The most

310 parsimonious explanation of many of the examples of pre-imaginal conditioning

311 reported in Drosophila involves a 'chemical legacy' of odoriferous particles on the

312 outside of the pupa that are detected on adult emergence, altering the activity of the

313 adult olfactory system and inducing a change in behavior (Barron 2001). We cannot

314 exclude this possibility, but the levels of cVA used in our experiments are far lower than

315 the levels of substances such as menthol which are traditionally used in pre-imaginal

316 conditioning experiments, particles of which are present on the pupa. Furthermore, the

317 pre-imaginal conditioning effect observed here will have a different neurobiological basis

318 than classical learning. 
320 Neither of the receptors that are currently known to detect cVA (Or65a and Or67d) are 321 expressed in larvae (Fishilevich et al. 2005), indicating that cVA must be perceived by 322 an as-yet unidentified larval receptor. Moreover, we found no evidence of attraction to 323 any dose of cVA in larval olfactory tests (Fig.S6). If conditioning works through altered 324 activity of larval neural circuits, conserved through metamorphosis and affecting adult neurons, by definition the unidentified stimuli that are involved with cVA in producing the conditioning effect must be detected by larvae.

The plasticity of pheromonally-induced courtship suppression we describe here in male flies contrasts with the widespread assumption that pheromones induce stereotypical and unconditional behaviors (Wyatt 2015). Modulation of an innate pheromonal response occurs in $C$. elegans, where early exposure to the repellent pheromone asc$\Delta 9$ increases adult responses to this substance through increased expression of the odr-2 glycosylated phosphatidylinositol (GPI)-linked signaling gene (Hong et al. 2017).

334 Given that cVA is found in a range of Drosophila species (Hedlund et al. 1996;

335 Symonds \& Wertheim 2005), conditioning may also affect male courtship inhibition in 336 these species. Equally, the widespread behavioral effects of cVA on both sexes of $D$. melanogaster may be based on conditioning, and thereby susceptible to showing greater levels of variability than is currently assumed.

340 The variation in the responses shown by males derived from eggs laid 1 and 10 days after mating, which correlates with the level of cVA on these eggs, reveals an unexpected source of phenotypic variability in the behavior of Drosophila siblings. Males derived from eggs laid by females 10 days after mating showed no cVA-based courtship

344 suppression, unlike their brothers derived from eggs laid 1 day after mating. The population-level variability in the courtship suppression phenotype produced by this effect would mean that some males would reduce their courtship of mated females, whereas others would not. 
349 An adaptationist explanation of the courtship suppression phenotype needs to be 350 nuanced to take this into account: it is not sufficient to suggest that by suppressing 351 courtship following contact with cVA, Drosophila males avoid 'wasteful' courtship that 352 does not lead to direct fitness benefits. Flies derived from eggs that are born at a longer 353 interval after mating will tend not to show this effect: there must be some advantage 354 underlying the need for conditioning rather than a labelled line effect whereby cVA 355 suppresses courtship under all circumstances. One possible explanation for such 356 plasticity effects is that they may enable rapid phenotypic adaptation in a changing 357 environment (Laland et al. 2015). In nature, such variation is not always relevant. 358 Depending upon the concentration and availability of potential sex partners, 359 D. melanogaster females lose most of the cVA that has been transferred to them after $36024 \mathrm{~h}$ and re-mate within 10 days post mating (Singh et al. 2002). However, the situation

361 may be different if fewer conspecific males are present. In this case, there may be an 362 advantage to not suppressing courtship when populations are small and unmated 363 females are extremely rare - persistent courtship may eventually lead to a female re364 mating (Smith et al. 2017; Yapici et al. 2008). 
365

366

367

368

369

370

371

372

373

374

375

376

377

378

379

380

381

382

383

384

385

386

387

388

389

390

391

392

393

394

395

\section{2}

\section{CONCLUSION}

Besides its involvement in the well-known male courtship suppression phenomenon, cVA is known for its multiple roles in D. melanogaster (Ejima 2015). Nowadays, all studies dealing with its roles in Drosophila chemical communication considered that responses to this compound are stereotypic and unconditional. We show that adult male courtship suppression by cVA is modulated by exposure to cVA - conjointly with some other substances - during the larval stage. Our findings, together with studies of chemical conditioning in vertebrates (Bett \& Hinch 2015; Coureaud et al. 2006; Hauser et al. 2017) and Drosophila females (Flaven-Pouchon et al. 2014) suggest that pheromonal responses may be less stereotypical than hitherto suspected, and that developmentally-determined plasticity may play an important role in naturally-occurring variation in chemical communication that may not be readily identified in the laboratory. Next step will be to evaluate if - and how - such a conditioning effect also affects the other Drosophila behavioural responses to cVA. Furthermore, our finding opens the road to the study of how conditioning affects nervous system development, and its evolutionary significance.

ACKNOWLEGMENTS: Jérôme Cortot, Mouhamadou Sall and Rhassane Asgassou are thanked for their help with flies.

\section{REFERENCES}

2012. XLSTAT 2012, Data analysis and statistics with Microsoft Excel. Paris, France: Addinsoft.

Barron AB. 2001. The Life and Death of Hopkins' Host-Selection Principle. Journal of Insect Behavior 14:725-737.

Barron AB, and Corbet SA. 1999. Preimaginal conditioning in Drosophila revisited. Animal Behaviour 58:621-628. 10.1006/anbe.1999.1169 
396 Bartelt RJ, Jackson LL, and Schaner AM. 1985a. Ester Components Of Aggregation

397

398

399

400

401

402

403

404

405

406

407

408

409

410

411

412

413

414

415

416

417

418

419

420

421

422

423

424

425

426 Pheromone Of Drosophila-Virilis (Diptera, Drosophilidae). Journal of Chemical Ecology 11:1197-1208.

Bartelt RJ, Schaner AM, and Jackson LL. 1985b. Cis-Vaccenyl Acetate As An Aggregation Pheromone In Drosophila-Melanogaster. Journal of Chemical Ecology 11:1747-1756.

Bett NN, and Hinch SG. 2015. Attraction of migrating adult sockeye salmon to conspecifics in the absence of natal chemical cues. Behavioral Ecology 26:11801187. 10.1093/beheco/arv062

Butterworth FM. 1969. Lipids of Drosophila: a newly detected lipid in the male. Science 163:1356-1357.

Clyne P, Grant A, O'Connell R, and Carlson JR. 1997. Odorant response of individual sensilla on the Drosophila antenna. Invertebrate Neuroscience 3:127-135.

Coureaud G, Moncomble AS, Montigny D, Dewas M, Perrier G, and Schaal B. 2006. A pheromone that rapidly promotes learning in the newborn. 16:1956-1961. 10.1016/j.cub.2006.08.030

Couto A, Alenius M, and Dickson BJ. 2005. Molecular, anatomical, and functional organization of the Drosophila olfactory system. Current Biology 15:1535-1547. 10.1016/j.cub.2005.07.034

Das S, Trona F, Khallaf MA, Schuh E, Knaden M, Hansson BS, and Sachse S. 2017. Electrical synapses mediate synergism between pheromone and food odors in Drosophila melanogaster. Proceedings of the National Academy of Sciences of the United States of America 114:E9962-E9971. 10.1073/pnas.1712706114.

Datta SR, Vasconcelos ML, Ruta V, Luo S, Wong A, Demir E, Flores J, Balonze K, Dickson BJ, and Axel R. 2008. The Drosophila pheromone cVA activates a sexually dimorphic neural circuit. Nature 452:473-477.

Ejima A. 2015. Pleiotropic actions of the male pheromone cis - vaccenyl acetate in Drosophila melanogaster. J Comp Physiol A 201:927-932.

Farine JP, Habbachi W, Cortot J, Roche S, and Ferveur JF. 2017. Maternallytransmitted microbiota affects odor emission and preference in Drosophila larva. Sci Rep 7. DOI: 10.1038/s41598-017-04922-z 
427 Fernandez MP, Chan YB, Yew JY, Billeter JC, Dreisewerd K, Levine JD, and Kravitz

428

429

430

431

432

433

434

435

436

437

438

439

440

441

442

443

444

445

446

447

448

449

450

451

452

453

454

455

EA. 2010. Pheromonal and behavioral cues trigger male-to-female aggression in Drosophila. PLoS Biology 8:e1000541. 10.1371/journal.pbio.1000541

Fernandez MP, and Kravitz EA. 2013. Aggression and courtship in Drosophila: pheromonal communication and sex recognition. Journal of comparative physiology A, Neuroethology, sensory, neural, and behavioral physiology 199:1065-1076. 10.1007/s00359-013-0851-5

Fishilevich E, Domingos Al, Asahina K, Naef F, Vosshall LB, and Louis M. 2005. Chemotaxis behavior mediated by single larval olfactory neurons in Drosophila. Current biology : CB 15:2086-2096. 10.1016/j.cub.2005.11.016

Flaven-Pouchon J, Garcia T, Abed-Vieillard D, Farine J-P, Ferveur J-F, and Everaerts C. 2014. Transient and Permanent Experience with Fatty Acids Changes Drosophila melanogaster Preference and Fitness. PLoS One 9. 10.1371/journal.pone.0092352

Greenspan RJ, and Ferveur JF. 2000. Courtship in Drosophila. Annual Review of Genetics 34:205-232.

Guiraudie-Capraz G, Pho DB, and Jallon JM. 2007. Role of the ejaculatory bulb in biosynthesis of the male pheromone cis-vaccenyl acetate in Drosophila melanogaster. Integr Zool 2:89-99. 10.1111/j.1749-4877.2007.00047.x

Hauser WJ, Westley PAH, Kerkvliet C, and Dudiak N. 2017. Homing of Pacific salmon to a marine release site: a case study of the Homer Spit Fishing Hole, Alaska. Northwest Science 91:314-323. doi.org/10.3955/046.091.0310

Hedlund K, Bartelt RJ, Dicke M, and Vet LEM. 1996. Aggregation pheromones of Drosophila immigrans, D-phalerata, and D-subobscura. Journal of Chemical Ecology 22:1835-1844.

Hong M, Ryu L, Ow MC, Kim J, Je AR, Chinta S, Huh YH, Lee KJ, Butcher RA, Choi H, Sengupta P, Hall SE, and Kim K. 2017. Early pheromone experience modifies a synaptic activity to influence adult pheromone responses of $C$. elegans. Current Biology 27:3168-3177. 
456 Houot B, Bousquet F, and Ferveur JF. 2010. The consequences of regulation of desat1

457

458

459

460

461

462

463

464

465

466

467

468

469

470

471

472

473

474

475

476

477

478

479

480

481

482

483

484 expression for pheromone emission and detection in Drosophila melanogaster. Genetics 185:1297-1309. 10.1534/genetics.110.117226

Jallon JM, Antony C, and Benamar O. 1981. An Anti-Aphrodisiac Produced By Drosophila-Melanogaster Males And Transferred To Females During Copulation. Comptes Rendus de l'Academie des Sciences Serie III Sciences de la Vie 292:1147-1149.

Karlson P, and Butenandt A. 1959. Pheromones (ectohormones) in insects. Annual Review of Entomology

4:39-58. doi.org/10.1146/annurev.en.04.010159.000351

Kohl J, Ostrovsky AD, Frechter S, and Jefferis GS. 2013. A bidirectional circuit switch reroutes pheromone signals in male and female brains. Cell 155:1610-1623. doi: 10.1016

Kurtovic A, Widmer A, and Dickson BJ. 2007. A single class of olfactory neurons mediates behavioural responses to a Drosophila sex pheromone. Nature 446:542-546.

Laland KN, Uller T, Fellman MW, Sterelny K, Muller GB, Moczek A, Jablonka E, and Odling-Smee J. 2015. The extended evolutionary synthesis: its structure, assumptions and predictions. Proc R Soc B 282:20151019. 10.1098/rspb.2015.1019

Lasbleiz C, Ferveur JF, and Everaerts C. 2006. Courtship behaviour of Drosophila melanogaster revisited. Animal Behaviour 72:1001-1012.

Laturney M, and Billeter JC. 2016. Drosophila melanogaster females restore their attractiveness after mating by removing male anti-aphrodisiac pheromones. . Nature Communication 7:12322 10.1038/ncomms12322

Lebreton S, Trona F, Borrero-Echeverry F, Bilz F, Grabe V, Becher PG, Carlsson MA, Nässel DR, Hansson BS, Sachse S, and Witzgall P. 2015. Feeding regulates sex pheromone attraction and courtship in Drosophila females. . Sci Rep 5:13132. 10.1038/srep13132 
485 Liu W, Liang X, Gong J, Yang Z, Zhang YH, Zhang JX, and Rao Y. 2011. Social

486

487

488

489

490

491

492

493

494

495

496

497

498

499

500

501

502

503

504

505

506

507

508

509

510

511

512

513

514

515 regulation of aggression by pheromonal activation of Or65a olfactory neurons in Drosophila. Nature Neuroscience 14:896-902. 10.1038/nn.2836

Manning A. 1967. Pre-imaginal conditioning in Drosophila. Nature 216:338-340.

Peng J, Chen S, Büsser S, Liu H, Honegger T, and Kubli E. 2005. Gradual release of sperm-bound sex-peptide controls female postmating behavior in Drosophila. Current Biology 15:207-223.

Robertson JL, and Preisler HK. 1992. Pesticide bioassays with Arthropods. Boca Raton, FL: CRC Press / Taylor \& Francis Group.

Ruta V, Datta SR, Vasconcelos ML, Freeland J, Looger LL, and Axel R. 2010. A dimorphic pheromone circuit in Drosophila from sensory input to descending output. Nature 468:686-690. 10.1038/nature09554

Schaner AM, Bartell RJ, and Jackson LL. 1987. (z)-II-Octadecenyl acetate, an aggregation pheromone in Drosophila simulans. Journal of Chemical Ecology 13:1777-1786.

Siegel RW, and Hall JC. 1979. Conditioned responses in courtship behavior of normal and mutant Drosophila. Proc Natl Acad Sci U S A 76:3430-3434.

Singh SR, Singh BN, and Hoenigsberg HF. 2002. Female remating, sperm competition and sexual selection in Drosophila. Genetics and Molecular Research 1:178-215.

Smith DT, Clarke NVE, Boone JM, Fricke C, and Chapman T. 2017. Sexual conflict over remating interval is modulated by the sex peptide pathway. Proc $R \operatorname{Soc} B$ 284: . 0.1098/rspb.2016.2394

Svetec N, and Ferveur JF. 2005. Social experience and pheromonal perception can change male-male interactions in Drosophila melanogaster. Journal of Experimental Biology 208:891-898.

Symonds MR, and Wertheim B. 2005. The mode of evolution of aggregation pheromones in Drosophila species. Journal of Evolutionary Biology 18:12531263. 10.1111/j.1420-9101.2005.00971.x

Tachibana SI, Touhara K, and Ejima A. 2015. Modification of male courtship motivation by olfactory habituation via the GABAA receptor in Drosophila melanogaster. PLoS One 10(8). doi:10.1371/journal.pone.0135186 
516 Thorpe WH. 1939. Further studies on pre-imaginal olfactory conditioning in insects. .

517

518 Tukey JW. 1977. Exploratory Data Analysis. Reading, Mass: Addison-Wesley Pub. Co. .

519 Tully T, Cambiazo V, and Kruse LT. 1994. Memory through metamorphosis in normal and mutant Drosophila. Journal of Neuroscience 14:68-74.

van der Goes van Naters W, and Carlson JR. 2007. Receptors and neurons for fly odors in Drosophila. Current Biology 17:606-612.

Wang L, Han X, Mehren J, Hiroi M, Billeter JC, Miyamoto T, Amrein H, Levine JD, and Anderson DJ. 2011. Hierarchical chemosensory regulation of male-male social interactions in Drosophila. Nature Neuroscience 14:757-762. 10.1038/nn.2800

Wertheim B, van Baalen EJ, Dicke M, and Vet LE. 2005. Pheromone-mediated aggregation in nonsocial arthropods: an evolutionary ecological perspective. Annual Review of Entomology 50:321-346. 10.1146/annurev.ento.49.061802.123329

Wyatt TD. 2015. How animals communicate via pheromones. American Scientist 103:114-121.

Yapici N, Kim YJ, Ribeiro C, and Dickson BJ. 2008. A receptor that mediates the postmating switch in Drosophila reproductive behaviour. Nature 451:33-37. doi:10.1038/nature06483

Zawistowski S, and Richmond RC. 1986. Inhibition Of Courtship And Mating Of Drosophila-Melanogaster By The Male-Produced Lipid, Cis-Vaccenyl Acetate. Journal of Insect Physiology 32:189-192. 
538 FIGURE LEGENDS

539

540 Figure 1:

541 Experimental procedure.

542 At day 0 (D0), male and female 4 day old WT1 flies were paired for $3 \mathrm{~h}$ and mated

543 females then transfered into egg-laying devices were allowed to lay eggs one day (+1D)

544 or 10 days after mating (+10D). For control experiments, $+1 \mathrm{D}$ and $+10 \mathrm{D}$ eggs, washed

545 and unwashed were placed on plain food. +1D washed eggs were deposited on plain

546 food either seeded with eggs from a second wild-type strain (+WT2 eggs) or on cVA-

547 rich food $(+\mathrm{cVA})$; other +1D washed eggs were covered with a solution of cVA and

548 placed on plain food. All focal male flies derived from these egg treatments were tested

549 in a double-deck chamber. Each male was paired with a beheaded virgin 4-day-old

550 WT1 female in the upper part of the chamber while a cVA dose was placed in the lower 551 part.

552

553 Figure 2:

554 Amount of cVA detected on washed eggs.

555 Amount (ng/egg) of cVA detected on eggs laid one day after mating (+1D) either control

$556(n=19)$ or washed with a $10 \%$ ethanol solution ("Wash"; $n=20)$. Amounts were

557 significantly different at $p \leq 10^{-4}$. Amounts are shown as box-and-whisker plots indicating

558 the 25th and 75th percentiles (boxes), the median (line within box) and the limits

559 (whiskers) beyond which values were considered anomalous. ${ }^{* * *}: p<0.001$

560

561 Figure 3:

562 Behavioral effect of cis-vaccenyl acetate (cVA) manipulation on eggs.

563 The courtship of focal males paired with beheaded females was measured for $10 \mathrm{~min}$, 564 with different cVA doses (0-2000ng). We noted the frequency of males courting (\%; $\mathbf{A}$,

565 C) and the total proportion of time spent courting (courtship index; B, D; the

566 corresponding courtship latencies are shown on Fig.S2). (A, B) We first compared the

567 courtship of control focal males (filled bars or circles) and males derived from washed

568 eggs (empty bars or diamonds) raised on plain food ( $n=15-91)$. (C, D) Using three cVA 
569 doses $(0,350,600 \mathrm{ng})$, we compared the courtship performance of males derived from

570 control and washed eggs with those of males derived from washed eggs placed on food

571 with WT2 eggs (light gray bars) or cVA-rich food (15ng/mm3; dark gray bars). Courtship

572 indices (D) are shown as box-and-whisker and significant differences are indicated by

573 different letters (for example, "a" differs from "b" but not from "ab"; $n=20-107$ ).

574 Corresponding values for courtship latency are shown in Fig.S4

575

576 Figure 4:

577 Post-mating effect on cVA conditioning.

578 (A) Comparison of cVA on eggs laid by the same females either one day (+1D) or 10

579 days (+10D) after mating ( $n=12$ and 10, respectively). (B) Courtship indices were

580 compared in males derived from $+1 \mathrm{D}$ and $+10 \mathrm{D}$ eggs tested with either 0 or $600 \mathrm{ng} \mathrm{cVA}$

$581(n=49-144) .{ }^{* * *}: p<0.001 ;{ }^{* *}: p<0.01$; ns: non-significant. For more details, see Fig.1.

582 Corresponding values for courtship frequency and latency are shown in Fig.S2.

583

584 Figure 5:

585 Identifying a critical period for cVA conditioning

586 Bars and box-plots show the courtship frequency (top) and courtship index (bottom) of

587 focal males derived from +1D eggs. These eggs were either control (filled bars) or were

588 washed at different periods after egg-laying $(+10,+20,+48,+72 \mathrm{~h}$; empty bars $)$ and then

589 placed on plain food. Males derived from these eggs were tested either with 600ng cVA

590 (A) or without cVA (B). For more details see Figs. 1 and 3. $n=10-45$. Corresponding

591 values for courtship latency are shown in Fig.S3.

592

593 Figure 6:

594 Effect of synthetic cVA on eggs.

595 +1D control eggs (filled bars and box-plots) were compared with washed eggs (empty

596 bars and box plots), and washed eggs covered with a cVA solution (shaded bars and

597 box-plots). All eggs were placed on plain food. We measured (A) the amount of cVA on

598 eggs ( $n=19$ for control, $n=8$ for Wash and $n=12$ for Wash $+c V A$ ), and (B) the courtship 599 index of derived males tested with 0 or 600 ng cVA $(n=61-203)$. For more details see 
600 Figs.1 and 5. Corresponding values for courtship frequency and latency are shown in 601 Fig.S5. 


\section{SUPPLEMENTARY FIGURE LEGENDS}

603

604 Figure S1:

605 Amount of cVA detected on and inside flies.

606 Box plots indicate the amount of cVA ( $\mathrm{ng} / \mathrm{fly}$ ) detected on virgin and recently mated

607 male and female flies ( $n=9-17)$. We used solvent extraction over two periods: the 20

608 min-long extraction revealed the cVA present on the fly cuticle, whereas the 24h-long

609 solvent extraction (at $40^{\circ} \mathrm{C}$ ) allowed us to extract internal cVA. For more details see

610 Fig.3.

611

\section{Figure S2:}

613 Post-mating effect.

614 Courtship frequency $(\mathbf{A})$ and latency $(B)$ were measured in focal males derived from

$615+1 \mathrm{D}$ and $+10 \mathrm{D}$ eggs in the presence of 0 or $600 \mathrm{ng} \mathrm{cVA}$. For more details see Figs. 1

616 and 4.

617

618 Figure S3:

619 Identification of a critical period.

620 Courtship latency was measured in focal males derived from control eggs (filled bars) or

621 from eggs washed at different times after egg-laying ( $A E L ;+10,+20,+48,+72$ h; empty

622 bars) with 600ng cVA (A) or no cVA (B). For more details see Figs.1 and 5.

623

624 Figure S4:

\section{Egg and food manipulation.}

626 Courtship latency was measured in focal males each paired with a beheaded female in

627 the presence of 0,350 or $600 \mathrm{ng}$ cVA. Focal males were derived from +1D eggs either

628 with no treatment (filled bars), or from washed eggs raised either on plain food (empty

629 bars), or on food seeded with WT2 eggs (light gray bars), or on cVA-rich food. For more

630 details see Fig.3. 


\section{Figure S5:}

\section{Effect of cVA added on eggs.}

633 Courtship frequency $(\mathbf{A})$ and latency $(B)$ were measured in focal males dervived from

634 control +1D eggs (filled bars), or of washed +1D eggs (empty bars), some of which were 635 covered with a synthetic cVA solution (shaded bars). All eggs were placed on plain food 636 and resulting males were tested with 0 or 600ng cVA. For more details see Figs. 1 and 6376.

638

639 Figure S6:

640 Larval responses to cVA

641 Individual larvae produced by +1D (A) or +10D (B) control eggs were placed on an agar 642 plate with two food sources at opposite sides. These were either plain food (PF) or PF 643 with the addition of $1 \mathrm{ng}-10 \mu \mathrm{g}$ of cVA. $\mathrm{N}=50$ for each comparison. The first food source 644 reached by each larva was noted. There were no significant differences in response to 645 cVA, nor were there any differences between +1D or +10D larvae.

646

647 Figure S7:

648 Cuticular hydrocarbons on eggs.

649 Comparison of total $\mathrm{CHs}$ amount on eggs laid by the females either one day (+1D) or 10 650 days $(+10 \mathrm{D})$ after mating ( $n=10 ; 50$ pooled eggs were extracted for each female). For 651 more details, see Fig. 1. 


\section{Figure 1 (on next page)}

Experimental procedure.

At day 0 (D0), male and female 4 day old WT1 flies were paired for $3 \mathrm{~h}$ and mated females then transfered into egg-laying devices were allowed to lay eggs one day (+1D) or 10 days after mating (+10D). For control experiments, $+1 \mathrm{D}$ and $+10 \mathrm{D}$ eggs, washed and unwashed were placed on plain food. +1D washed eggs were deposited on plain food either seeded with eggs from a second wild-type strain (+WT2 eggs) or on cVA-rich food (+CVA); other +1D washed eggs were covered with a solution of cVA and placed on plain food. All focal male flies derived from these egg treatments were tested in a double-deck chamber. Each male was paired with a beheaded virgin 4-day-old WT1 female in the upper part of the chamber while a cVA dose was placed in the lower part. 


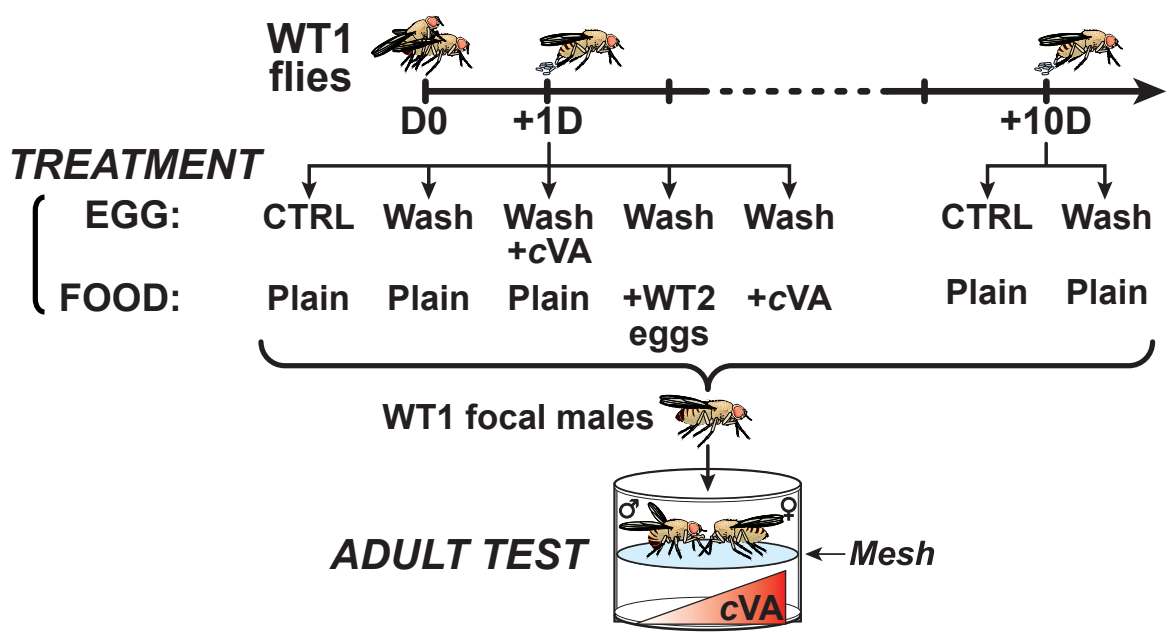


Figure 2 (on next page)

Amount of cVA detected on washed eggs.

Amount (ng/egg) of cVA detected on eggs laid one day after mating (+1D) either control $(n=19)$ or washed with a $10 \%$ ethanol solution ("Wash"; $n=20$ ). Amounts were significantly different at $p \leq 10^{-4}$. Amounts are shown as box-and-whisker plots indicating the 25th and 75th percentiles (boxes), the median (line within box) and the limits (whiskers) beyond which values were considered anomalous. ${ }^{* * *}: p<0.001$ 


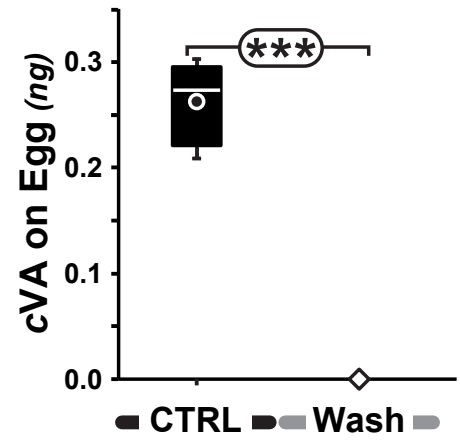

Egg treatment 


\section{Figure 3 (on next page)}

Behavioral effect of cis-vaccenyl acetate (cVA) manipulation on eggs.

The courtship of focal males paired with beheaded females was measured for $10 \mathrm{~min}$, with different cVA doses (0-2000ng). We noted the frequency of males courting (\%; $\mathbf{A}, \mathbf{C})$ and the total proportion of time spent courting (courtship index; B, D; the corresponding courtship latencies are shown on Fig.S2). (A,B) We first compared the courtship of control focal males (filled bars or circles) and males derived from washed eggs (empty bars or diamonds) raised on plain food ( $n=15-91)$. (C,D) Using three $\operatorname{CVA}$ doses $(0,350,600 n g)$, we compared the courtship performance of males derived from control and washed eggs with those of males derived from washed eggs placed on food with WT2 eggs (light gray bars) or CVA-rich food (15ng/mm3; dark gray bars). Courtship indices (D) are shown as box-and-whisker and significant differences are indicated by different letters (for example, "a" differs from "b" but not from "ab"; $n=20-107)$. Corresponding values for courtship latency are shown in Fig.S4. 
A

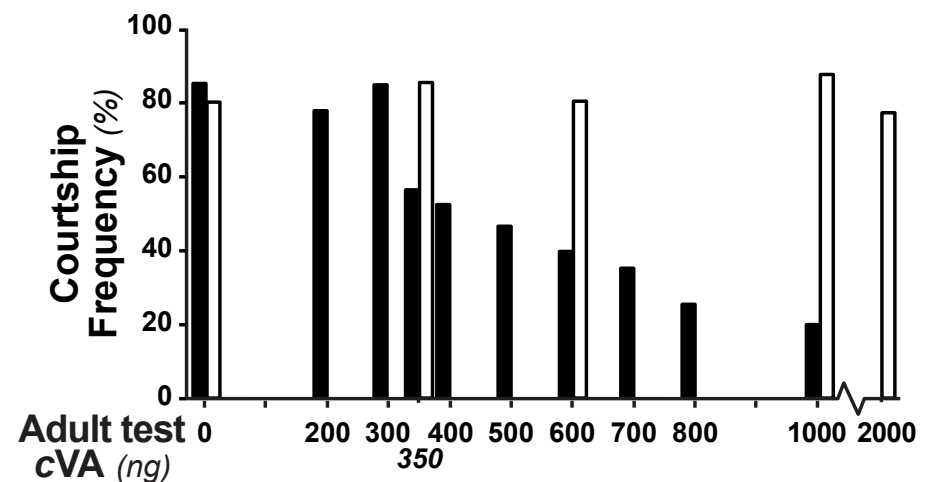

C

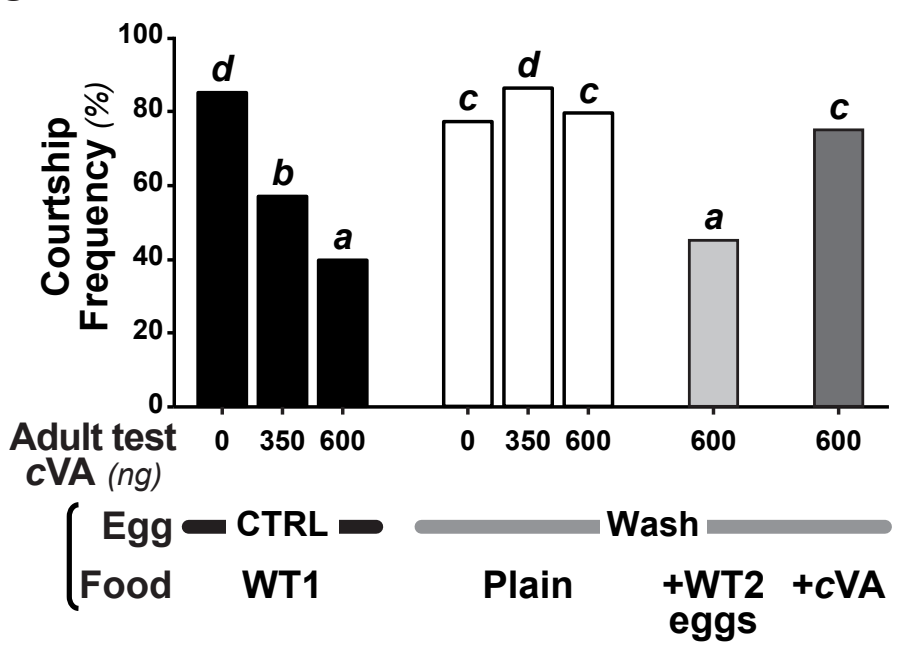

B
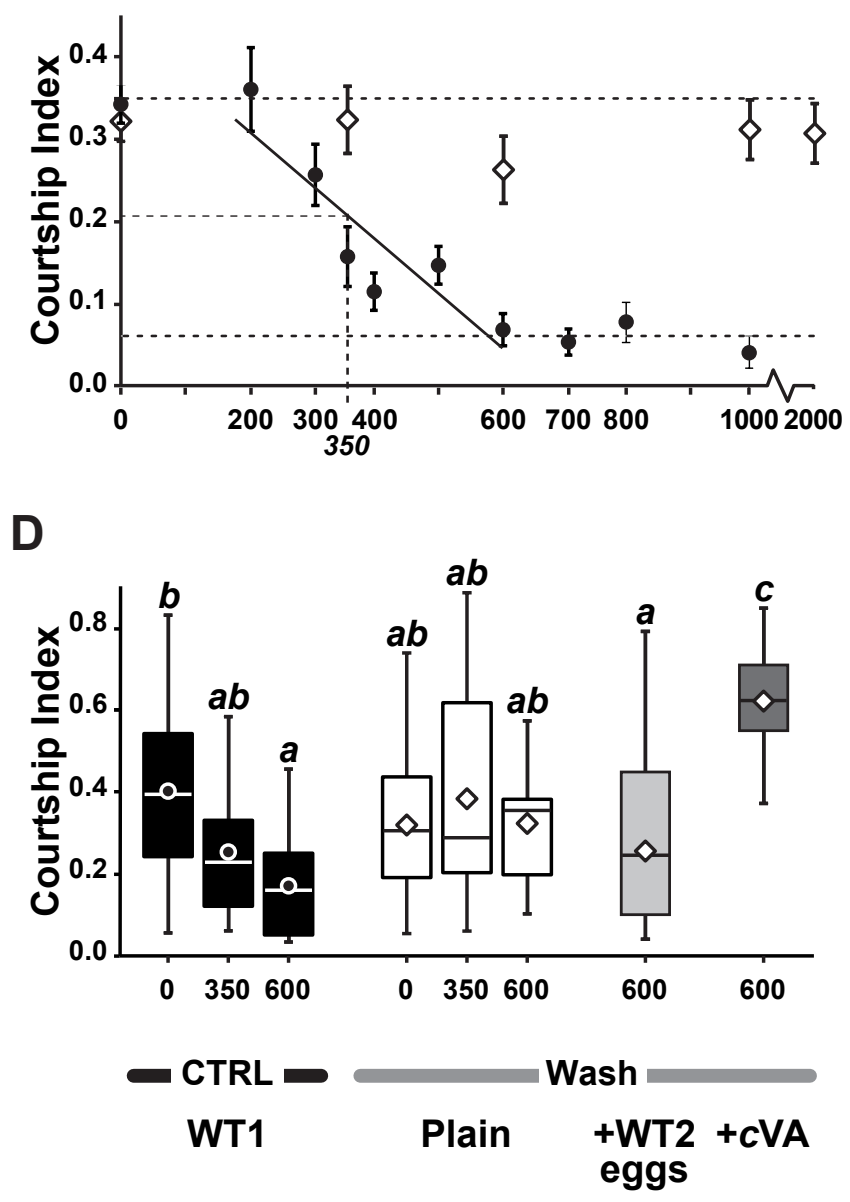
Figure 4 (on next page)

Post-mating effect on cVA conditioning.

(A) Comparison of CVA on eggs laid by the same females either one day (+1D) or 10 days (+10D) after mating ( $n=12$ and 10, respectively). (B) Courtship indices were compared in males derived from $+1 \mathrm{D}$ and $+10 \mathrm{D}$ eggs tested with either 0 or 600ng CVA ( $n=49-144)$. ***:p<0.001; **:p<0.01; ns: non-significant. For more details, see Fig.1. Corresponding values for courtship frequency and latency are shown in Fig.S2. 
a

PeerJ

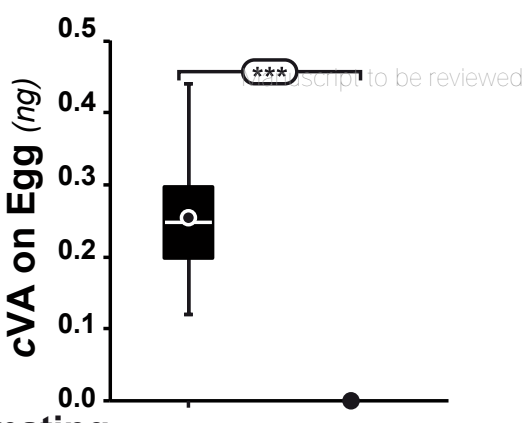

Post-mating egg layinge+1D $=$ +10D (days)

b

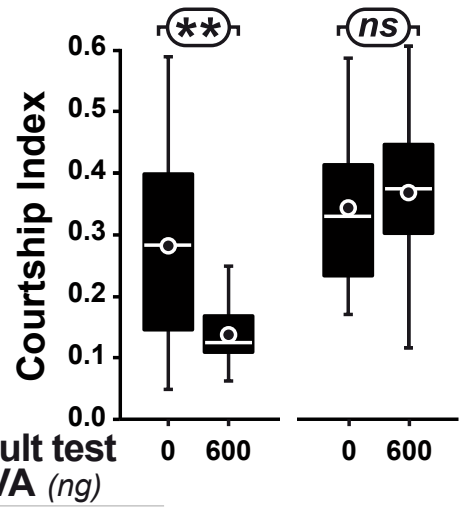

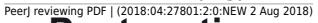

Post-mating egg laying $a+1 D=a+10 D$ D (days) 
Figure $\mathbf{5}$ (on next page)

Identifying a critical period for CVA conditioning.

Bars and box-plots show the courtship frequency (top) and courtship index (bottom) of focal males derived from $+1 \mathrm{D}$ eggs. These eggs were either control (filled bars) or were washed at different periods after egg-laying $(+10,+20,+48,+72 \mathrm{~h}$; empty bars $)$ and then placed on plain food. Males derived from these eggs were tested either with 600ng CVA (A) or without CVA (B). For more details see Figs. 1 and 3. $n=10-45$. Corresponding values for courtship latency are shown in Fig.S3. 
A

B
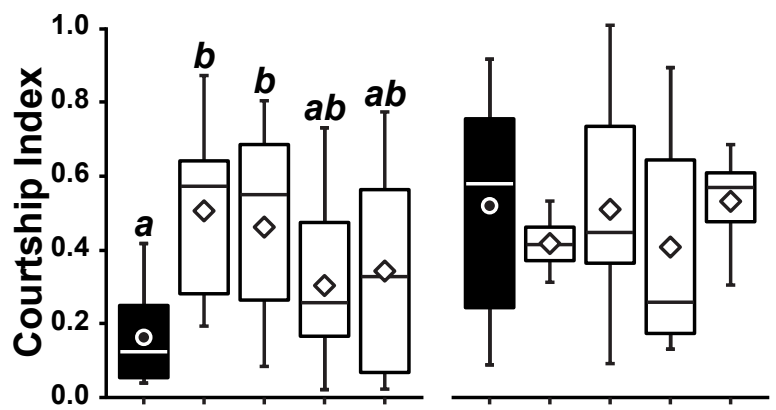

Egg was

䍃

Adult test

cVA (ng)

600 


\section{Figure 6 (on next page)}

\section{Effect of synthetic cVA on eggs.}

+1D control eggs (filled bars and box-plots) were compared with washed eggs (empty bars and box plots), and washed eggs covered with a cVA solution (shaded bars and box-plots). All eggs were placed on plain food. We measured (A) the amount of cVA on eggs ( $n=19$ for control, $n=8$ for Wash and $n=12$ for Wash $+c V A)$, and (B) the courtship index of derived males tested with 0 or 600ng cVA $(n=61-203)$. For more details see Figs. 1 and 5.

Corresponding values for courtship frequency and latency are shown in Fig.S5. 
a

PeerJ

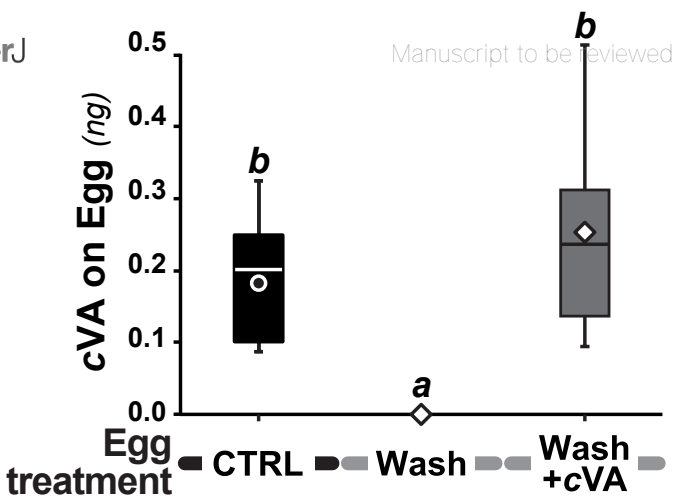

b

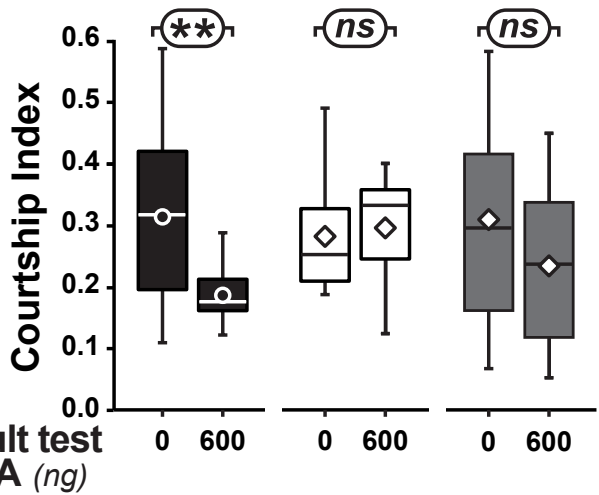

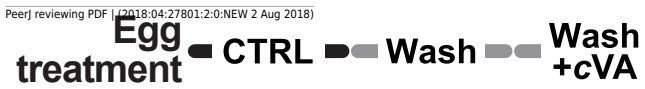

